\title{
LIFeS: Laboratório de Interação Flexível e Sustentável
}

\author{
Vânia Paula de Almeida Neris \\ Departamento de Computação (DC) \\ Universidade Federal de São Carlos (UFSCar) \\ São Carlos, Brasil \\ vania@dc.ufscar.br
}

\begin{abstract}
RESUMO
Este artigo apresenta o Laboratório de Interação Flexível e Sustentável (LIFeS). Este laboratório foi fundado em 2010 e está hospedado no Departamento de Computação da Universidade Federal de São Carlos. O objetivo principal do laboratório é avançar o estado da arte em Ciência e Engenharia da Computação, especialmente nos campos da Interação Homem-Computador. A pesquisa centra-se na identificação da diversidade dos usuários, incluindo aspectos emocionais, e no projeto e avaliação de soluções de interface de usuário flexíveis para vários dispositivos, plataformas e contextos como web, ubíquo e móvel. Mais recentemente, o laboratório vem investigando como projetar aplicações terapêuticas. Também se investiga como levar a sustentabilidade ao design e como os designers podem favorecer a sustentabilidade por meio de soluções computacionais.
\end{abstract}

\section{PALAVRAS-CHAVE}

Interfaces de Usuário Flexíveis, Emoções, Aplicações Terapêuticas, Sustentabilidade, Laboratório.

\section{INTRODUÇÃO}

O Laboratório de Interação Flexível e Sustentável (LIFeS), fundado em 2010, reúne pesquisadores interessados em avançar o estado da arte em computação, em particular em temas relacionados à Interação Humano-Computador (IHC). O laboratório, hospedado em uma sala de $20 \mathrm{~m} 2$ no Departamento de Computação da Universidade Federal de São Carlos, reúne alunos de computação da graduação e pós-graduação (mestrado e doutorado), além de contar com a colaboração de alunos e professores de outras áreas do conhecimento como Enfermagem, Gerontologia, Pedagogia e Educação Especial.

O LIFeS atua em ações de pesquisa e também de extensão, firmando parcerias com grupos da sociedade para intervenções de interesse na área de IHC. Até o momento, o grupo vem recebendo fomento da UFSCar, da CAPES, do CNPq e da FAPESP em bolsas e auxílios.

Permission to reproduce or distribute, in whole or in part, material extracted from this work, verbatim, adapted or remixed, as well as the creation or production from the content of such work, is granted without fee for non-commercial use, provided that the original work is properly credited.

IHC 2019 - TRILHA FÓRUM DOS GRUPOS DE PESQUISA, Outubro 21-25, 2019, Vitória, Brasil. In Anais Estendidos do XVIII Simpósio Brasileiro sobre Fatores Humanos em Sistemas Computacionais. Porto Alegre: SBC

(C) 2019 by the author(s), in accordance with the terms of the Creative Commons Attribution-NonCommercial 4.0 International Public License (CC BY-NC 4.0).

\section{PRINCIPAIS CONTRIBUIÇÕES DE PESQUISA}

O LIFeS1 iniciou suas atividades de pesquisa com especial interesse em interfaces de usuário flexíveis, isto é, aquelas que mudam seu comportamento em tempo de execução. Depois, passou a investigar também como inferir o estado emocional de um usuário e outras questões relacionadas. Mais recentemente tem investigado como conceber aplicações terapêuticas e aspectos de sustentabilidade na computação. Além desses, o LIFeS tem realizado pesquisas iniciais em composição musical algorítmica visando a produção sonora de acordo com padrões do ouvinte humano, considerando a diversidade de contextos associados a atores com objetivos particulares.

\section{Interfaces de usuário flexíveis}

Formalizado em 2010, o PLuRaL é um framework para o design de interfaces de usuário flexíveis, que visa apoiar designers desde a etapa de clarificação do problema de design até a materialização da solução de design $[9,10]$. Este trabalho, premiado no IHC2010, teve uma versão estendida publicada em periódico [11]. A partir dele, o laboratório inicia suas pesquisas no tema em diferentes plataformas e contextos de uso.

Jogos Digitais: No que se refere aos jogos, o laboratório tem especial contribuição em como organizar o sistema para que a flexibilidade da interface de usuário seja alcançada $[3,4,5]$. Um destaque aqui está para a proposição de diretivas para áudio games [4] que tem sido citada internacionalmente.

Dispositivos móveis e TV Digital: Os trabalhos com dispositivos móveis e flexibilidade focaram no público idoso. A formalização de um conjunto de normas para alteração, durante a interação, da interface de usuário de um celular foi premiada no IHC2011 [7] e estendida em periódico internacional [8]. Com TV, o LIFeS contribuiu com estudos com idosos e conteúdo complementar [18] e em como avaliar a interação [16].

\section{Aplicações terapêuticas}

A pesquisa sobre como conceber aplicações terapêuticas surge por um chamado de uma instituição de saúde. Ao se buscar na literatura, percebeu-se a necessidade de se investigar, com as lentes de IHC, como conceber tais sistemas. Em 2014, o LIFeS propõe e avalia um conjunto de passos para a clarificação desse problema de design [17]. Esse trabalho, premiado no IHC2014, teve uma versão estendida em periódico [19]. Em 2016, o

\footnotetext{
${ }^{1}$ www.dc.ufscar.br/lifes
} 
laboratório formaliza uma linguagem de modelagem da interação para esse domínio específico [6] e o jogo "Jogar Também Faz Bem!" é escolhido a melhor demonstração do IHC2016 [14]. Em 2018, o LIFeS formalizou uma abordagem para o design de jogos digitais terapêuticos, a SemTh $[23,24]$. A SemTh é uma abordagem semio-participativa que tem por objetivo apoiar diferentes partes interessadas durante o design de jogos digitais com finalidade terapêutica. Pode-se citar também a proposição de um editor que permitiu que pessoas em reabilitação de uso abusivo de álcool e drogas com baixo letramento e sem experiência no uso de computadores criassem jogos [26].

\section{Emoções em IHC}

$\mathrm{O}$ LIFeS foi um dos primeiros grupos de pesquisa em IHC no Brasil a investigar como considerar as emoções no design e avaliação de soluções computacionais. Em 2012, foram investigadas decisões de design ruins e o impacto delas nas emoções [22]. Em 2014, é publicada uma abordagem holística, pautada em diferentes métodos, para a avaliação de respostas emocionais de usuários [21]. Em 2015, o laboratório propõe um método analítico, de base Semiótica, para avaliação do impacto emocional de usuários [20] e o círculo de cores de Bianchi para apoio a escolha de cores considerando as emoções que se deseja evocar [2]. Em 2016, o grupo avalia métodos para inferência de emoções em ambientes ubíquos [1]. Em 2018, foram iniciados estudos que exploram a influência emocional da música com o usuário. Desenvolveu-se um algoritmo de recomendação de música que considera o estado emocional desejado do usuário [25]. Atualmente, o grupo executa o projeto Emoweb, com financiamento da Fundação de Amparo a Pesquisa do Estado de São Paulo (FAPESP \#15/24523-8).

\section{Sustentabilidade}

O laboratório participou da proposição do Desafio 1 do GrandIHC-Br que faz menção a importância de se investigar como considerar aspectos de sustentabilidade no design e também como desenvolver soluções computacionais que possam fomentar um comportamento mais sustentável nos usuários [12]. Nessas temáticas, o LIFeS realizou um revisão sistemática para saber como a comunidade de IHC mundial tem contribuído com esses temas [13] e também um levantamento de como a comunidade brasileira entende essas questões [15].

\section{CONSIDERAÇÕES FINAIS}

O LIFeS é ainda um laboratório novo, com menos de 10 anos de existência, mas que tem tentado colaborar com a comunidade de IHC por meio de pesquisas que visam auxiliar designers a melhor conceber e avaliar sistemas computacionais interativos, reconhecendo e respeitando a diversidade de usuários. Também se deve ressaltar a intenção dos pesquisadores em colaborar com outros grupos de pesquisa no Brasil.

Como pontos de melhoria para o laboratório, pode-se citar a necessidade de buscar mais financiamento para a pesquisa e colaborações com outros grupos de pesquisa em IHC no Brasil e também internacionais.

\section{AGRADECIMENTOS}

Agradeço às minhas orientadoras, Profa. Dra. Júnia Coutinho Anacleto (UFSCar) e Profa. Dra. Maria Cecília Calani Baranaukas
(UNICAMP), pela paciência e preciosos ensinamentos sobre a pesquisa em IHC. Agradeço também a todos os colegas de pesquisa dos laboratórios por onde passei, em particular pelo LIA (UFSCar), InterHAD (UNICAMP) e IRC (University of Reading Inglaterra). Agradeço imensamente a todos os alunos que já passaram pelo LIFeS, pelas trocas, contribuições e ensinamentos que me deixaram. Agradeço a todos os usuários - parceiros cocriadores que foram pacientes conosco e nos ajudaram a ver mais longe. Agradeço a todos os pesquisadores, de diversas áreas, que investigaram e contribuíram conosco. Agradeço às agências e demais fontes de financiamento. Por fim, agradeço a comunidade brasileira de IHC pelas valiosas contribuições e receptividade aos trabalhos do LIFeS.

\section{REFERÊNCIAS}

[1] Tatiana S. Alencar, et. al. Emotional Response Evaluation of users in Ubiquitous Environments: an Observational Case Study. In: 13th Advances in Computer Entertainment Technology Conference (ACE2016). Osaka. Proc. Article 18, 2016. 12p.

[2] Renata G. Bianchi, Vânia P. A. Neris. Com que cor eu vou? Um estudo sobre cores e emoções na Interação Humano-Computador. In: XIV Simpósio Brasileiro sobre Fatores Humanos em Sistemas Computacionais (IHC2015), Salvador.

[3] Franco E. Garcia, Neris, Vânia P. A. Neris. Design de Jogos Universais: Apoiando a Prototipação de Alta Fidelidade com Classes Abstratas e Eventos. In: XII Simpósio Brasileiro sobre Fatores Humanos em Sistemas Computacionais (IHC2013). Manaus. Anais, 2013. p. 82-91.

[4] Franco E. Garcia, Neris, Vânia P. A. Neris. Design Guidelines for Audio Games. In: 15th International Conference on Human-Computer Interaction (HCI2013). Las Vegas. LNCC, 2013. p. 229-238.

[5] Franco E. Garcia, Neris, Vânia P. A. Neris. A Data-Driven Entity-Component Approach to Develop Universally Accessible Games. In: 16th International Conference on Human-Computer Interaction (HCI2014). Creta. HumanComputer Interaction. Applications and Services. LNCC, 2014. p. 537-548.

[6] Franco E. Garcia, et al. An Interaction Modeling Language for Therapeutic Applications. In: XV Simpósio Brasileiro sobre Fatores Humanos em Sistemas Computacionais (IHC2016), São Paulo. Anais Article 32, 2016. 10p.

[7] Vinicius P. Gonçalves, et al. Interação de Idosos com Celulares: Flexibilidade para Atender a Diversidade. In: Simpósio Brasileiro sobre Fatores Humanos em Sistemas Computacionais (IHC2011). Porto de Galinhas. Anais, 2011.

[8] Vinicius P. Gonçalves, et al. Providing adaptive smartphone interfaces targeted at elderly people: an approach that takes into account diversity among the elderly. Universal Access in the Information Society (Print), v. 1, p. 1-21, 2015.

[9] Vânia P. A. Neris, Maria C. C. Baranauskas. Making Interactive Systems More Flexible: An Approach Based on Users Participation and Norms. In: Simpósio Brasileiro sobre Fatores Humanos em Sistemas Computacionais (IHC2010) Belo Horizonte. 2010.

[10] Vânia P. A. Neris, Maria C. C. Baranauskas. A Framework for Designing Flexible Systems. In: Proc. of IEEE International Conference on Systems_Man_and Cybernetics (SMC) - Human-Machine Systems Track, 2011, Anchorage.

[11] Vânia P. A. Neris, Maria C. C. Baranauskas. Designing tailorable software systems with the users' participation. Journal of the Brazilian Computer Society (Impresso), v. 18, Issue 3, pp 213-227. 2012

[12] Vânia P. A. Neris, et al. Futuro, Cidades Inteligentes e Sustentabilidade. GranDIHC-BR - Grandes Desafios de Pesquisa em Interação HumanoComputador no Brasil (2012), 16-18.

[13] Vânia P. A. Neris, et al. A Systematic Review about Sustainability and Aspects of Human-Computer Interaction. In: 16th International Conference on HumanComputer Interaction (HCI2014). Creta. LNCC, 2014.

[14] Diogo Nishikawa, et al. Se cuidar, cuidar de algo, se divertir e aprender fazem bem! - Demonstração de um jogo para apoiar o tratamento da depressão 2016 (Demonstração de software) no XV Simpósio Brasileiro sobre Fatores Humanos em Sistemas Computacionais (IHC2016). São Paulo. 2016.

[15] Renata R. Oliveira, et al. Percepções sobre Aspectos de Sustentabilidade na Computação. In: XV Simpósio Brasileiro sobre Fatores Humanos em Sistemas Computacionais (IHC2016). São Paulo. Anais, 2016.

[16] Kamila R. H. Rodrigues, et al. Heuristics for assessing emotional response of viewers during the interaction with TV programs. In: 16th International Conference on Human-Computer Interaction (HCI2014) Creta. LNCC, 2014. p. 577-588.

[17] Kamila R. H. Rodrigues, et al. Enriquecimento de Personas para Apoio ao Design de Aplicações Terapêuticas para a Saúde Mental. In: XIII Simpósio 
Brasileiro sobre Fatores Humanos em Sistemas Computacionais (IHC2014). Foz do Iguaçu. 2014.

[18] Kamila R. H. Rodrigues, et al. Interaction of the elderly viewer with additional multimedia content to support the appreciation of television programs. In: 15th International Conference on Human-Computer Interaction (HCI2013), Las Vegas. 2013. p. 227-236

[19] Kamila R. H. Rodrigues, et al. Personas-Driven Design for Mental Health Therapeutic Applications. SBC Journal on 3D Interactive Systems, v. 6, p. 1834, 2015.

[20] Maria C. S. Santos, Vânia P. A. Neris. A caminho de um método analítico para avaliação de respostas emocionais na interação humano-computador. In: XIV Simpósio Brasileiro sobre Fatores Humanos em Sistemas Computacionais, (IHC2015). Salvador.

[21] Rogério A. C. Xavier, Vânia P. A. Neris. A Hybrid Semantic Approach to Measure the Emotional Experience of Users. In: XIII Simpósio Brasileiro sobre Fatores Humanos em Sistemas Computacionais (IHC2014). Foz do Iguaçu. 2014.

[22] Rogério A. C. Xavier, et al. Decisões de design de interfaces ruins e o impacto delas na interação: um estudo preliminar considerando o estado emocional de idosos. In: Simpósio Brasileiro sobre Fatores Humanos em Sistemas Computacionais (IHC2012). Cuiabá. Anais, 2012. p. 127-136.

[23] Paula Maia de Souza, Kamila Rios da Hora Rodrigues, Vânia Paula de Almeida Neris. Towards a Semiotic-Based Approach to the Design of Therapeutic Digital Games. In: K. Liu, K. Nakata, W. Li, \& C. Baranauskas, editors, Digitalisation, Innovation, and Transformation, IFIP Advances in Information and Communication Technology, 53-62. Springer International Publishing. 2018.

[24] Paula Maia de Souza. Abordagem para o Design de Jogos Digitais Terapêuticos. 115 p. Dissertação de mestrado. Universidade Federal de São Carlos. São Carlos, SP. 2018.

[25] Willian Garcias de Assunção, Vania Paula de Almeida Neris. An algorithm for music recommendation based on the user's musical preferences and desire emotions. In Proceedings of the 17th International Conference on Mobile and Ubiquitous Multimedia (MUM), Slim Abdennadher and Florian Alt (Eds.) ACM, New York, NY, USA, 205-213, 2018.

[26] Garcia, F. E. ; Brandao, R.; Mendes, G. C. P. ; Neris, V.P.A . Able to Create, Able to (Self)Improve: How an Inclusive Game Framework Fostered SelfImprovement Through Creation and Play in Alcohol and Drugs Rehabilitation? In: IFIP TC.13 International Conference on Human-Computer Interaction (INTERACT), 2019, Paphos. Proceedings of the 17th IFIP TC.13 International Conference on Human-Computer Interaction. Berlim: Springer-Verlag, 2019. p. $1-10$ 